\title{
БЛОКИ ЦЕЛЫХ ПРОСТЫХ ЧИСЕЛ
}

\author{
Мазуркин Петр Матвеевич \\ Д-р техн. наук., проф., Поволжский государственный \\ технологический университет, г. Йошкар-Ола \\ E-mail: kaf_po@mail.ru \\ BLOCKS OF ENTIRE PRIMES \\ Mazurkin Peter Matveevich \\ Doctor of Engineering Science, \\ Volga State University of Technology, \\ Yoshkar-Ola, Republic of Mari El, Russian Federation
}

\begin{abstract}
Аннотация Двоичное разложение чисел образует геометрические блоки. Они зависят от приближения простого или целого простого числа к значениям двойки в степени, равной числу из натурального ряда. В итоге появляется строгая геометрия в ряду простых или целых простых чисел в виде блочной структуры. Эта структура получает отличительные признаки и показаны гармоники в положительной части ряда целых простых чисел. Статистически из ряда в 10 миллионов натуральных чисел доказаны закономерности роста мощности левого и правого реперов, как границ предыдущего и последующего от значений двойки в степени, у возрастающих по длине блоков в ряду целых простых чисел
\end{abstract}

Abstract Binary representations of numbers form a geometric blocks. They depend on the approximation of the simple or the whole of a prime number to the values of two to the power equal to the number of natural numbers. As a result, there is a strict geometry in a series of simple integers or primes in the form of block structure. This structure obtains features shown and harmonic positive integer number of prime numbers. Statistically, a series of 10 million integers proven capacity of growth of the left and right frames as boundaries from the previous and subsequent values of two in the extent, by increasing the length of blocks in the entire row primes

Ключевые слова: целые простые числа, положительная часть, двоичное представление, блочная структура, репера, особенности, волновая картина

Keywords: integer primes, positive part, the binary representation, the block structure, features, wave pattern

Введение. До наших публикаций [1-8] математики считали, что известные ряды простых чисел не обладают геометрическими свойствами. Гауссов ряд и специальных ряды простых чисел казались бессмысленным нагромождением чисел в виде непонятной «лестницы», по которой взбирается ведущий в одном из серии фильмов по истории математики. Сильный психологический барьер в понимании геометрического смысла возник, когда молодой Гаусс убрал из подаренной ему к 15-летнему возрасту таблицы простых чисел единицу. Тем самым была полностью отсечена система двоичного счисления из начала полного ряда простых чисел.

Наши исследования показали, что природа «считает» в двоичной системе счисления, а человек - в десятичной системе. Поэтому оказалось, что любой ряд простых чисел можно свернуть в двоичной системе счисления [4]. И только в этом случае появляется геометрия как самой гауссовой лестницы, так особенно его ступенек без треугольного основания этой лестницы (прироста простых чисел $[2,6])$.

Цель статьи - показать блочную структуру ряда целых простых чисел на положительной его части и дать закономерности количества (мощности) простых чисел в раду из 10 миллионов натуральных чисел. 
Квантование простого числа. Любое натуральное или целое число, включая и простые числа, можно представить как квантованный в двоичной системе счисления дискретный сигнал. Но квантование и формирование (генерация) простых чисел пока является неизвестным процессом.

При квантовании вся область значений сигнала разбивается на уровни, количество которых должно быть представлено в числах заданной разрядности. Расстояния между этими уровнями называется шагом квантования, и он для не разреженного (не специфического) ряда натуральных или целых чисел равен 1. Целые и положительные простые числа (далее простые числа ПЧ) являются специфическими рядами, причем с пока неизвестным переменным с возрастанием ряда ПЧ шагом квантования (длиной блока ПЧ). Число этих уровней (разрядов двоичной системы счисления) зависит от мощности квантуемого ряда простых чисел.

Известно давно, что для чисел, растущих по относительно простым законам, например, по степеням двойки, было бы, конечно, нелепо разыскивать экземпляр, превосходящий все известные. Для простых же чисел прилагаются громадные усилия, чтобы именно это и сделать. Простые числа как ряды подвергались только факторизации, т.е. разложению по множителям и числам с большими степенями двойки. Однако до сих пор не найдены пределы факторизации у ряда простых чисел.

Наши результаты показывают [7], что нужно делать разложение по слагаемым, причем эти слагаемые будут из двоичного представления числа из десятичной системы счисления. Именно такой подход позволяет вернуться математикам непосредственно к изучению самих рядов, а не заниматься законами роста количества простых чисел в разрядах десятичной системы счисления.

Вот это увлечение факторизацией, как мы полагаем, и не позволило математикам до нас применить двоичную систему для анализа не только множителей любого простого числа, но и квантованных в двоичной системе счисления слагаемых от любого натурального или целого числа, и, в частности, и любого простого числа. Разложение по слагаемым из двоичной системы счисления дает преимущества в наглядности геометрии.

Рассмотрим несколько основных свойств, присущих простым числам.

1. Любое простое число в известном гауссовом ряду $a(n)=2,3,5,7,11, \ldots$ содержит разряды $i_{2}=1,2, \ldots$ двоичной системы счисления и поэтому имеет после разложения на слагаемые составляющие

$$
a_{i_{2}}(n)=2^{i_{2}-1}
$$

где $a_{i_{2}}(n)$ - квантованная по уровням $i_{2}$ двоичной системы часть ПЧ.

2. Любое целое простое число равно сумме составляющих из двоичной системы счисления с учетом матрицы инцидентности

$$
a(n)=\sum_{i_{2}=1}^{\infty} \xi\left(i_{2}, n\right) a_{i_{2}}(n)=\sum_{i_{2}=1}^{\infty} \xi\left(i_{2}, n\right) 2^{i_{2}-1},
$$

где $\xi\left(i_{2}, n\right)$ - матрица инцидентности, причем всегда $\xi\left(i_{2}, n\right)=0 \vee 1$. 
Блок простых чисел. Вычислительные эксперименты показали, что мощность ряда принимается исходя из полноты блоков матрицы инцидентности. Для примера берем блок № 11 из положительной части ряда простых чисел [4], имеющий параметры: $n=(173,309), a(n)=(1031,2039), i_{2}=(1,11)$.

Расчеты по блоку № 11 даны в таблице 1 и были выполнены по формуле

$$
z_{2}=a_{1}-a_{2} \cos \left(\pi a(n) /\left(a_{3}+a_{4} a(n)^{a_{5}}\right)-a_{6}\right),
$$

где $z_{2}$ - двоичное число, в пределах от 0 до 1 как вещественное число,

$a(n)=2,3,5,7,11, \ldots$ - гауссов ряд простых чисел,

$a_{1} \ldots a_{6}$ - параметры статистической модели (3).

Закон (3) в общем виде [2] позволил доказать гипотезу Римана [5, 6].

Влияние ПЧ на двоичное число в блоке № 11 по разрядам двоичной системы

\begin{tabular}{|c|c|c|c|c|c|c|c|c|c|}
\hline \multirow{2}{*}{$\begin{array}{c}\text { Ра3- } \\
\text { ряд } \\
i_{2}\end{array}$} & \multirow{2}{*}{$\begin{array}{c}\text { Часть } \\
a_{i_{2}}(n)\end{array}$} & \multirow{2}{*}{\begin{tabular}{|c|} 
Средне- \\
статист. \\
$\bar{z}_{2 \phi}$
\end{tabular}} & \multicolumn{6}{|c|}{ Параметры статистической модели (3) двоичного числа } & \multirow{2}{*}{$\begin{array}{c}\text { Коэфф. } \\
\text { коррел. } \\
r\end{array}$} \\
\hline & & & $a_{1}$ & $a_{2}$ & $a_{3}$ & $a_{4}$ & $a_{5}$ & $a_{6}$ & \\
\hline 1 & 1 & 1 & 0.5 & -0.5 & 0 & 0 & 0 & 0 & 1 \\
\hline 2 & 2 & 0.51825 & 0.5 & 0.5 & 2 & 0 & 0 & 1.59217 & 1 \\
\hline 3 & 4 & 0.51825 & 0.5 & 0.70711 & 4 & 0 & 0 & 1.57080 & 1 \\
\hline 4 & 8 & 0.53285 & 0.50079 & 0.64897 & 8.00054 & 0 & 0 & -4.72553 & 0.9251 \\
\hline 5 & 16 & 0.48175 & 0.50339 & -0.64642 & 15.99613 & 0 & 0 & 4.82479 & 0.9069 \\
\hline 6 & 32 & 0.40876 & 0.50997 & 0.63517 & 32.02910 & 0 & 0 & 1.46990 & 0.8975 \\
\hline 7 & 64 & 0.51825 & 0.52117 & 0.63090 & 66.31876 & -0.00066974 & 1 & 0.090540 & 0.9066 \\
\hline 8 & 128 & 0.51095 & 0.50345 & 0.61806 & 129.7168 & $8.62532 \mathrm{e}-5$ & 1.11225 & 0.94630 & 0.9132 \\
\hline 9 & 256 & 0.48175 & 0.49203 & 0.64200 & 266.3384 & $1.85033 \mathrm{e}-5$ & 1.52406 & 0.73950 & 0.9147 \\
\hline 10 & 512 & 0.48905 & 0.50536 & 0.61721 & 682.0366 & -0.34387 & 0.64381 & -0.35596 & 0.9291 \\
\hline 11 & 1024 & 1 & 0.5 & -0.5 & 0 & 0 & 0 & 0 & 1 \\
\hline
\end{tabular}

Сравнение показало значимость в блоках последовательности простых чисел в ряду, а порядковый номер имеет только вспомогательное значение (заметим, что полнота порядкового номера будет соблюдена только при принятии полного ряда $\mathrm{N}=\{0,1,2,3,4,5,6, \ldots\}$ натуральных чисел). Нуль нельзя игнорировать, так как не будут достоверными статистические закономерности.

Поэтому основным показателем, вне зависимости от типа ряда простых чисел, становится среднестатистическое (только не среднеарифметическое) двоичное число, а переменной - само простое число.

В столбце $i_{2}=1$ из таблицы 1 по формуле (3) получаем двоичное число $\mathrm{z}_{2}=a_{1}-a_{2}=0,5-(-0,5)=1$.

Такое же соотношение будет для столбца $i_{2}=11$. Тогда для блока № 11 все точки на столбцах первого и 11-го разрядов двоичного счисления будут равны $z_{2}=1$. Последовательно рассматривая все блоки ряда простых чисел можно показать, что для первого столбца (правая граница двоичной записи простых чисел) условие $z_{2}=1$ будет соблюдаться на всей бесконечной полуоси некритичных простых чисел $a(n)=(3, \infty)$. По мере роста простого числа левая граница будет смещаться скачком от блока к блоку $[2,4]$. 
Геометрия двоичного числа. По модели (3) для разных разрядов $i_{2}$ двоичного числа по блоку простых чисел № 11 построены графики. Критическая линия Римана расположена на второй вертикали [2].

Если не учитывать первый и последний разряды двоичной системы, то по таблице 1 наиболее близко к рациональному корню 1/2 дзэта-функции Римана по вещественным значениям находится разряд $i_{2}=2$ (рис. 1). Дисперсия $s=$ 0,00000676 мала, поэтому коэффициент корреляции $r$ равен единице. Тогда критическая линия Римана есть вертикаль $i_{2}=2$ разложения ряда ПЧ и ЦПЧ.

Из данных таблицы 1 для блока № 11 получаем модель (рис. 1) вида

$$
z_{2}=1 / 2-1 / 2 \cos (\pi a(n) / 2-1,59217),
$$

в которой вещественное число 0,5 заменили на дробное число $1 / 2$.

С увеличение мощности ряда простых чисел будет стремление 1,59217 $\rightarrow \pi / 2$ и поэтому формула (4) получает вид закона

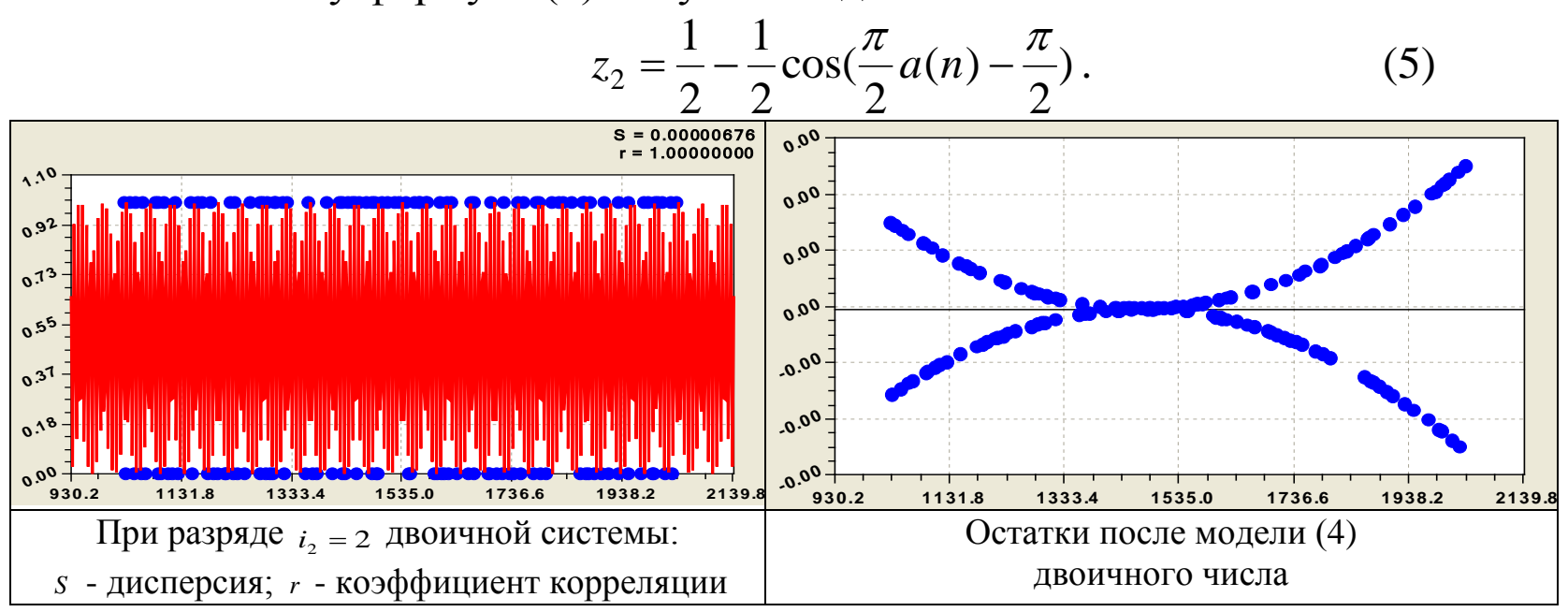

Рисунок 1. Графики статистической модели двоичного числа на втором разряде

Для разряда $i_{2}=3$ двоичного числа получено (рис. 2) уравнение

$$
z_{2}=1 / 2-0,70711 \cos (\pi a(n) / 4-1,57080) \text {, }
$$

которое при большой мощности ряда простых чисел преобразуется к виду

$$
z_{2}=\frac{1}{2}-\frac{\sqrt{2}}{2} \cos \left(\frac{\pi}{4} a(n)-\frac{\pi}{2}\right) .
$$

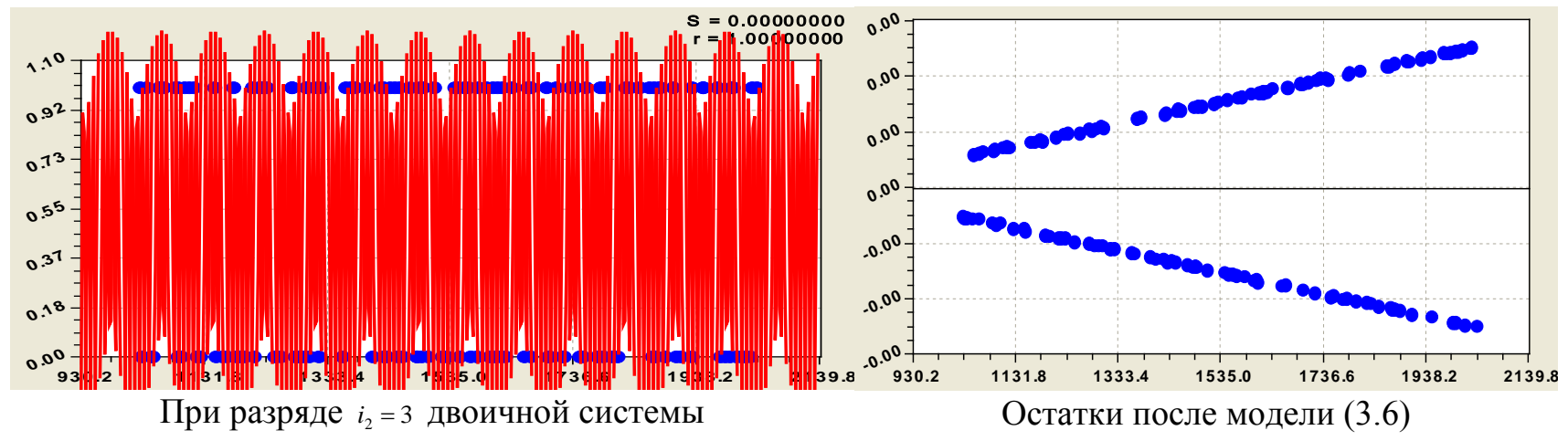

Рисунок 2. Графики модели двоичного числа на третьем разряде двоичного счисления

Хотя остатки после (6) приближаются к нулю, однако, как показывают графики (рис. 2), погрешность модели растет с увеличением простого числа. 
Поэтому должны появиться новые составляющие к модели (6).

Для разряда $i_{2}=4$ двоичного числа у 11-го блока ПЧ (рис. 3 ) есть формула

$$
z_{2}=0,50079-0,64897 \cos (\pi a(n) / 8,00054+4,72553),
$$

в которой значения параметров уже далеки от действительного корня 1/2.

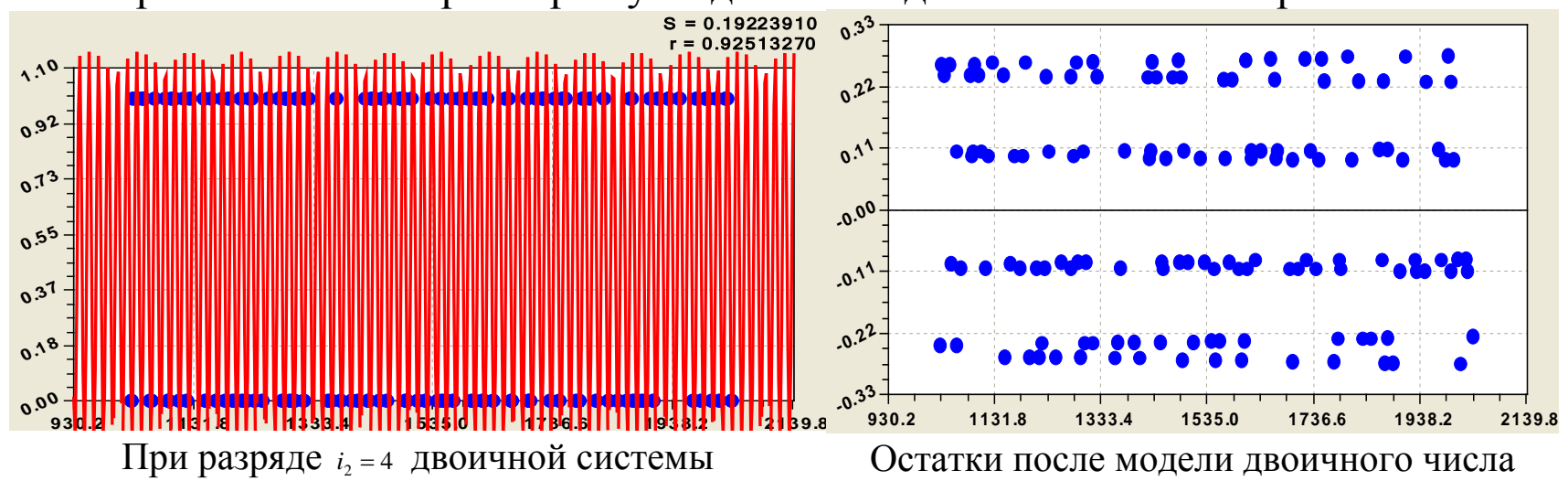

Рисунок 3. Графики модели двоичного числа на четвертом разряде двоичной системы

Поэтому только вторая вертикаль становится критической линией Римана.

Варианты структуры целых простых чисел (ЦПЧ). Блоки выделяются через преобразование целых простых чисел из десятичной в двоичную систему.

Для целых простых чисел любой мощности есть варианты структуры:

1) симметричный конечномерный ряд ЦПЧ с центром симметрии

\begin{tabular}{|c|c|c|c|c|c|c|c|c|c|c|c|c|c|c|c|}
\hline$Z$ & -7 & -6 & -5 & -4 & -3 & -2 & -1 & 0 & 1 & 2 & 3 & 4 & 5 & 6 & 7 \\
\hline$P_{Z}$ & -13 & -11 & -7 & -5 & -3 & -2 & -1 & 0 & 1 & 2 & 3 & 5 & 7 & 11 & 13 \\
\hline
\end{tabular}

2) симметричный конечномерный ряд ЦПЧ без центра симметрии

\begin{tabular}{|c|c|c|c|c|c|c|c|c|c|c|c|c|}
\hline$Z$ & -7 & -6 & -5 & -4 & -3 & & & 3 & 4 & 5 & 6 & 7 \\
\hline$P_{Z}$ & -13 & -11 & -7 & -5 & -3 & & & 3 & 5 & 7 & 11 & 13 \\
\hline
\end{tabular}

3) асимметрично влево или вправо ориентированные конечномерные ряды ЦПЧ с центром симметрии

\begin{tabular}{|c|c|c|c|c|c|c|c|c|c|c|c|c|c|c|c|}
\hline$Z$ & -9 & -8 & -7 & -6 & -5 & -4 & -3 & -2 & -1 & 0 & 1 & 2 & 3 & 4 & 5 \\
\hline$P_{Z}$ & -19 & -17 & -13 & -11 & -7 & -5 & -3 & -2 & -1 & 0 & 1 & 2 & 3 & 5 & 7 \\
\hline
\end{tabular}

\begin{tabular}{|c|c|c|c|c|c|c|c|c|c|c|c|c|c|c|c|}
\hline$Z$ & -5 & -4 & -3 & -2 & -1 & 0 & 1 & 2 & 3 & 4 & 5 & 6 & 7 & 8 & 9 \\
\hline$P_{Z}$ & -7 & -5 & -3 & -2 & -1 & 0 & 1 & 2 & 3 & 5 & 7 & 11 & 13 & 17 & 19 \\
\hline
\end{tabular}

4) левый (отрицательный) и правый (положительный) асимметричные ряды простых чисел

\begin{tabular}{|c|c|c|c|c|c|c|c|c|}
\hline$Z$ & -7 & -6 & -5 & -4 & -3 & -2 & -1 & -0 \\
\hline$P_{Z}$ & -13 & -11 & -7 & -5 & -3 & -2 & -1 & -0 \\
\hline
\end{tabular}

\begin{tabular}{|l|l|l|l|l|l|c|c|}
\hline 0 & 1 & 2 & 3 & 4 & 5 & 6 & 7 \\
\hline 0 & 1 & 2 & 3 & 5 & 7 & 11 & 13 \\
\hline
\end{tabular}

Четвертый тип симметричен для отрицательных и положительных ПЧ.

Закономерности структурных вариантов. Для сравнения рассмотрены примеры симметричных и асимметричных рядов ЦПЧ с количеством пар простых чисел 20 (табл. 2).

Для 20 пар ЦПЧ по основному закону распределения вдоль оси $Z$ получена (рис. 4) формула 


$$
P_{Z 20}=2,91568 Z \text {. }
$$

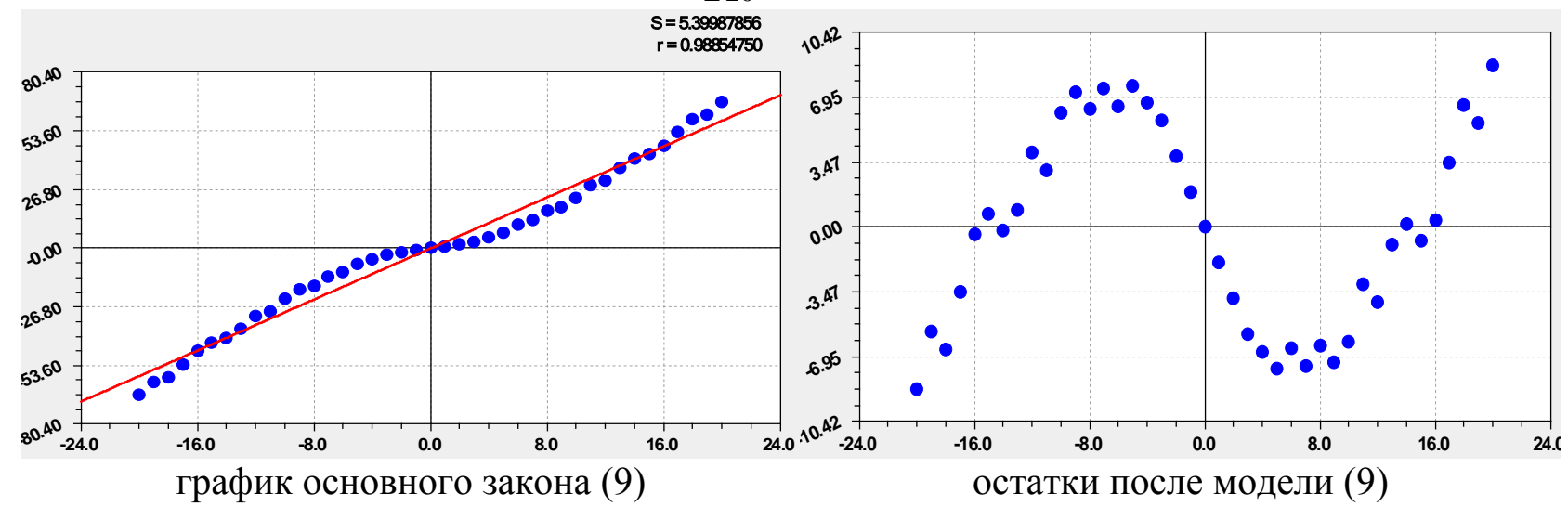

Рисунок 4. Графики формулы (9.1) распределения 20 пар ЦПЧ по шкале целых чисел

Асимметрия влево, например $n^{-}=20$ и $n^{+}=10$, дается (рис. 5) формулой

$$
P_{\text {Z20Л } 10 \Pi}=-1,40524+2,73831 Z \text {. }
$$

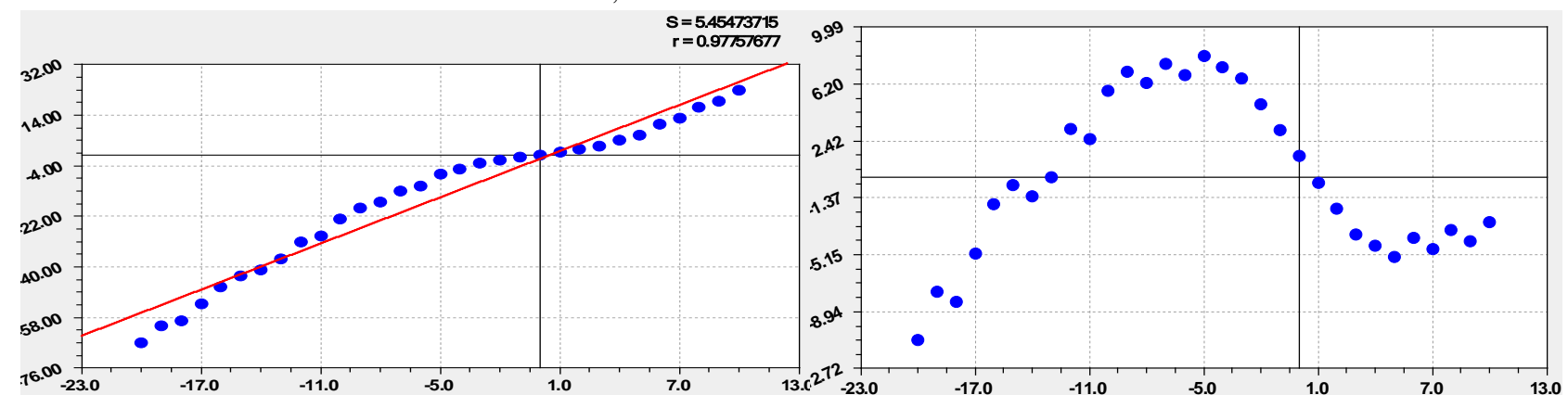

график по модели (10) левой асимметрии

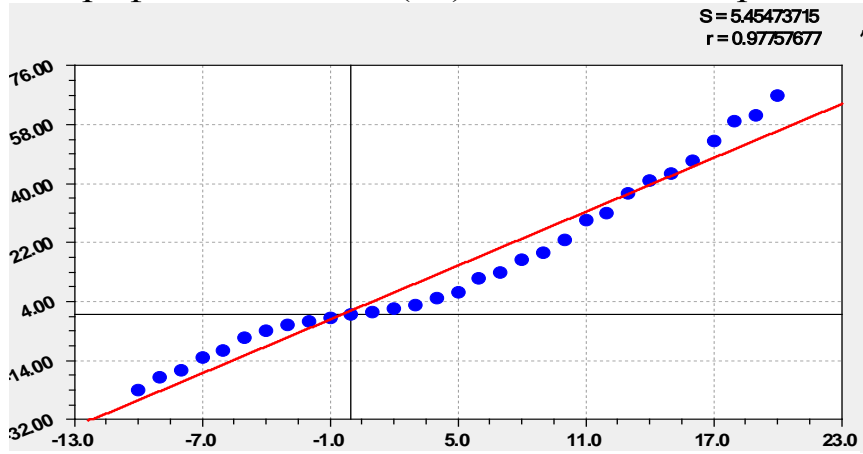

график по модели (11) правой асимметрии остатки после модели (10)

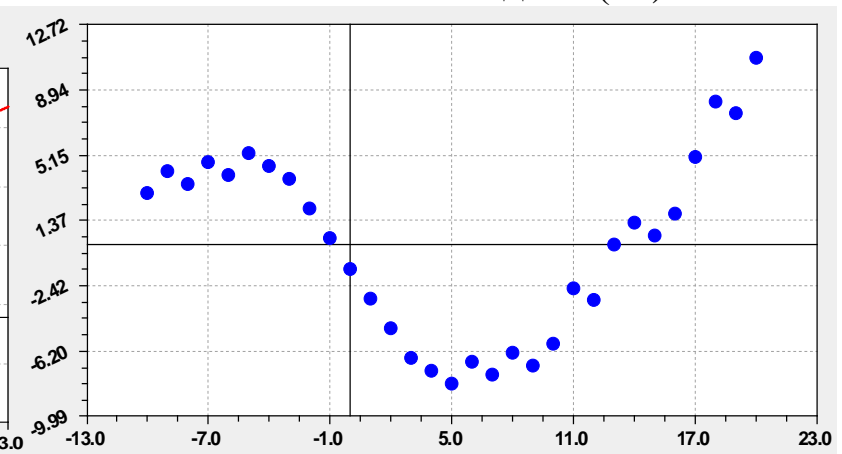

остатки после модели (11)

Рисунок 5. Графики распределения асимметричных пар ЦПЧ по шкале целых чисел

При асимметрии вправо, например $n^{-}=10$ и $n^{+}=20$, получается (рис. 5):

$$
P_{\text {Z10л }, 20 \Pi}=1,40524+2,73831 Z \text {. }
$$

Сравнение формул (10) и (11) показывает, что левая и правая асимметрия отличаются друг от друга только знаками первой составляющей, появляющейся в виде постоянного члена перед законом распределения членов симметричного ряда целых простых чисел. Доведем асимметрию до предела, когда начало (положительные ПЧ) или конец (для отрицательных ПЧ) определяются началом координат оси целых чисел.

Для простых чисел (полный ряд [2, 4]) 0, 1, 2, 3, 5, 7, ... (рис. 6) получим

$$
P_{+Z}=0,720694 Z_{+}{ }^{1,515400} \text {. }
$$




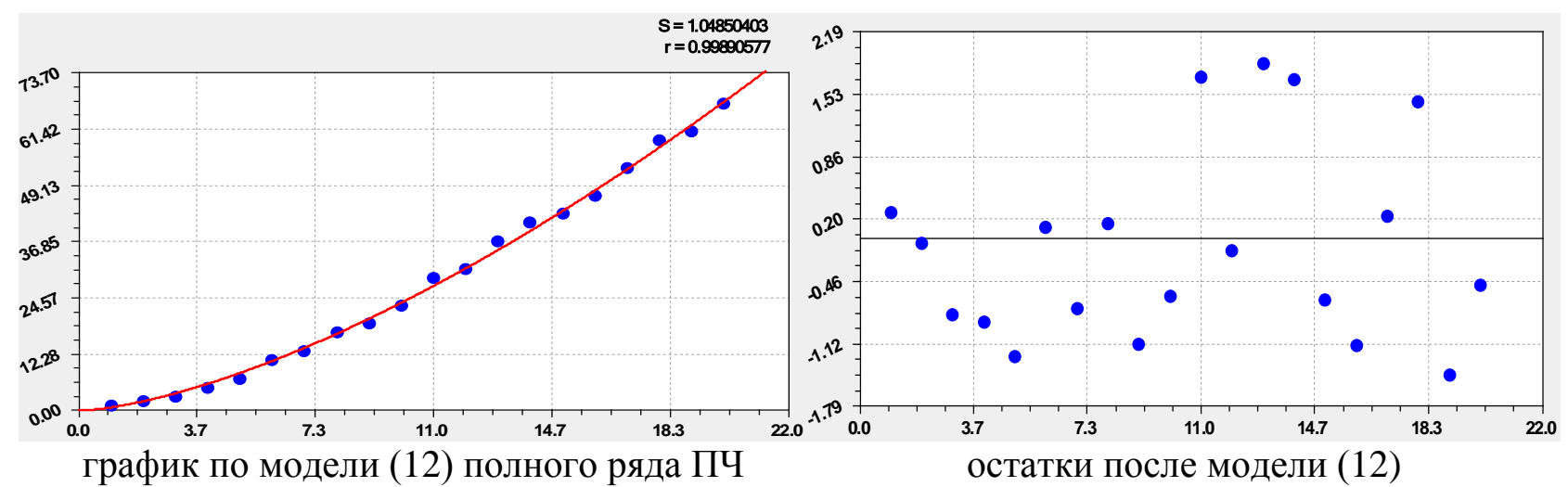

Рисунок 6. Распределение положительных ПЧ по правой шкале целых чисел

Из-за свойства симметричности, получаем модель

$$
P_{-Z}=-0,720694\left(-Z_{-}\right)^{1,515400} .
$$

Для прямой идентификации закономерностей на отрицательной полуоси целых чисел применим целочисленные степени в показательном законе

$$
P_{Z}=a Z^{b=1,2,4,6, \ldots} \text {. }
$$

По методике $[9,10]$ перебором $b=1,2,4,6, \ldots$ находим степень в (14) с максимальным коэффициентом корреляции: $b=1 r=0,9698 ; 2-0,9834 ; 4-0,7819$.

Наилучшим является степень 2 (рис. 7) и подтверждается теория Гаусса по применению 2 в законе нормального распределения $y=a \exp \left(-b(x-c)^{\wedge} 2\right)$, т.е.

$$
P_{-Z}=-0,18470 Z_{-}^{2} \text {. }
$$

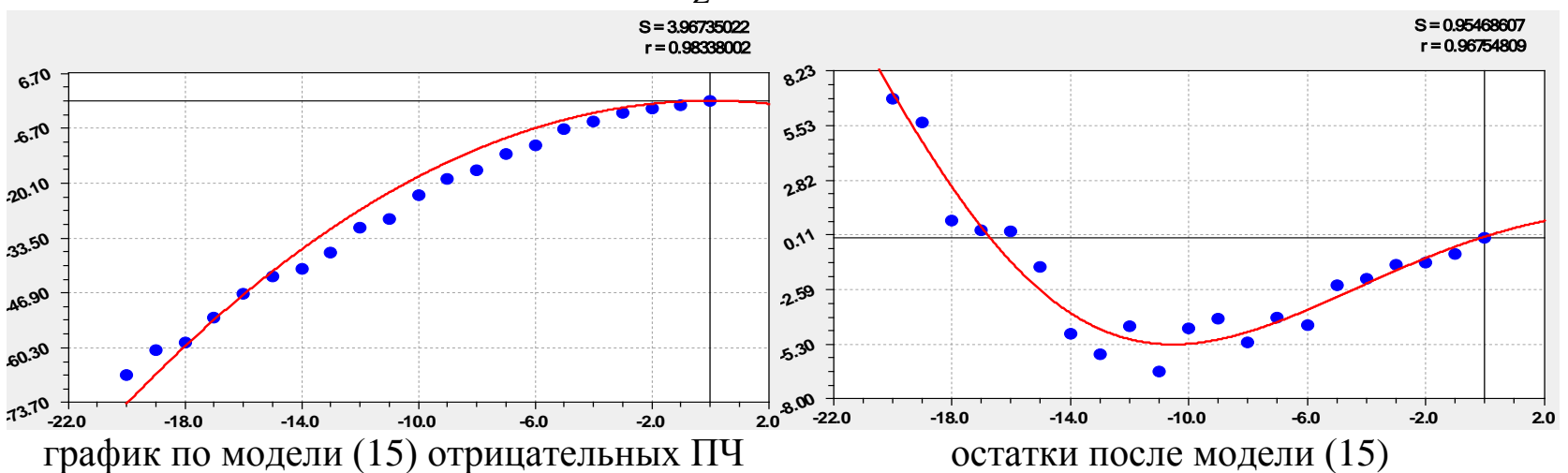

Рисунок 7. Распределение отрицательных простых чисел по левой шкале целых чисел

Совместная параметризация дает (рис. 8) двухчленное уравнение вида $P_{-Z 20}=-0,199339 Z_{-}{ }^{2}+1,566783 \exp \left(-0,110277 Z_{-}\right) \sin (\pi / 1,512178-0,103841)$.(16)

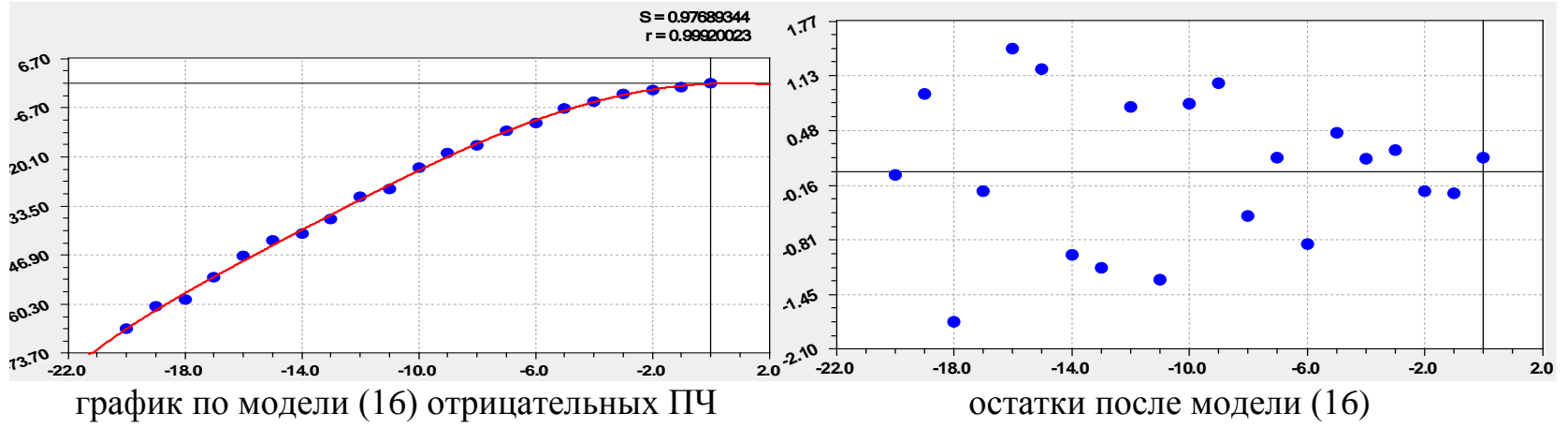

Рисунок 8. Распределение 20 отрицательных ПЧ по левой шкале целых чисел 
Первый отрицательный член показывает квадратичную функцию, а второй спад по амплитуде при постоянном периоде колебания $2 \times 1,51218=3,02436$.

Двоичное число симметричных рядов. Данные для идентификации приведены в таблице 2.

Влияние ряда ЦПЧ $P_{Z 20}$ на двоичное число $z_{2}$ по вертикали $i_{Z}^{P}=2$ идет по формуле

$$
z_{2}=-0,5 \sin \left(\pi P_{Z 20-3} / 2\right) .
$$

Здесь исключены три ЦПЧ $(-1,0,1)$ ядра центра симметрии из-за того, что во второй вертикали в этих клетках находятся тривиальные нули.

Коэффициент корреляции (17) равен чуть больше 0,7: мешается начало с простым числом 2 ряда ГауссаРимана $(2,3,5,7, \ldots)$.

После исключения из ЦПЧ центра симметрии, на двух симметричных относительно оси $Z$ некритичных рядах $\pm(3,5,7, \ldots)$, получилась

$z_{2}=\cos \left(\pi P_{Z 20-5} / 2+0,58903\right) .(1$

8)

Коэффициент корреляции стал равным 0,7454. Тогда получается, что тривиальные нули всегда делят вертикали на обособленные части. Поэтому нужно моделировать по частям некрити-

ческие половины рядов $P_{Z= \pm(3,5,7,11, \ldots)}$ (рис. 9):
Разложения 20 пар ЦПЧ и прироста

Таблийа 2

\begin{tabular}{|c|c|c|c|c|c|c|c|c|c|c|c|c|}
\hline \multirow{4}{*}{$\begin{array}{c}\text { Целое } \\
\text { число } \\
Z\end{array}$} & \multirow{4}{*}{$\begin{array}{c}\text { Прос- } \\
\text { тое } \\
\text { число } \\
P_{Z}\end{array}$} & \multirow{4}{*}{$\begin{array}{c}\text { При- } \\
\text { рост } \\
p_{Z}\end{array}$} & \multicolumn{7}{|c|}{ Разряд $i_{z}^{P}$ двоичный } & \multicolumn{3}{|c|}{ Разряд $i_{z}^{p}$} \\
\hline & & & 7 & 6 & 5 & 4 & 3 & 2 & 1 & 3 & 2 & 1 \\
\hline & & & \multicolumn{7}{|c|}{ Часть $P_{i Z}=2^{\wedge}\left(i_{Z}^{P}-1\right)$} & \multicolumn{3}{|c|}{$p_{i Z}=2^{\wedge}\left(i_{Z}^{p}-1\right)$} \\
\hline & & & 64 & 32 & 16 & 8 & 4 & 2 & 1 & 4 & 2 & 1 \\
\hline-20 & -67 & 6 & -1 & 0 & 0 & 0 & 0 & -1 & -1 & 1 & 1 & 0 \\
\hline-19 & -61 & 2 & & -1 & -1 & -1 & -1 & 0 & -1 & & 1 & 0 \\
\hline-18 & -59 & 6 & & -1 & -1 & -1 & 0 & -1 & -1 & 1 & 1 & 0 \\
\hline-17 & -53 & 6 & & -1 & -1 & 0 & -1 & 0 & -1 & 1 & 1 & 0 \\
\hline-16 & -47 & 4 & & -1 & 0 & -1 & -1 & -1 & -1 & 1 & 0 & 0 \\
\hline-15 & -43 & 2 & & -1 & 0 & -1 & 0 & -1 & -1 & & 1 & 0 \\
\hline-14 & -41 & 4 & & -1 & 0 & -1 & 0 & 0 & -1 & 1 & 0 & 0 \\
\hline-13 & -37 & 6 & & -1 & 0 & 0 & -1 & 0 & -1 & 1 & 1 & 0 \\
\hline-12 & -31 & 2 & & & -1 & -1 & -1 & -1 & -1 & & 1 & 0 \\
\hline-11 & -29 & 6 & & & -1 & -1 & -1 & 0 & -1 & 1 & 1 & 0 \\
\hline-10 & -23 & 4 & & & -1 & 0 & -1 & -1 & -1 & 1 & 0 & 0 \\
\hline-9 & -19 & 2 & & & -1 & 0 & 0 & -1 & -1 & & 1 & 0 \\
\hline-8 & -17 & 4 & & & -1 & 0 & 0 & 0 & -1 & 1 & 0 & 0 \\
\hline-7 & -13 & 2 & & & & -1 & -1 & 0 & -1 & & 1 & 0 \\
\hline-6 & -11 & 4 & & & & -1 & 0 & -1 & -1 & 1 & 0 & 0 \\
\hline-5 & -7 & 2 & & & & & -1 & -1 & -1 & & 1 & 0 \\
\hline-4 & -5 & 2 & & & & & -1 & 0 & -1 & & 1 & 0 \\
\hline-3 & -3 & 1 & & & & & & -1 & -1 & & & 1 \\
\hline-2 & -2 & 1 & & & & & & -1 & 0 & & & 1 \\
\hline-1 & -1 & 1 & & & & & & & -1 & & & 1 \\
\hline 0 & 0 & 1 & & & & & & & 1 & & & 1 \\
\hline 1 & 1 & 1 & & & & & & & 1 & & & 1 \\
\hline 2 & 2 & 1 & & & & & & 1 & 0 & & & 1 \\
\hline 3 & 3 & 2 & & & & & & 1 & 1 & & 1 & 0 \\
\hline 4 & 5 & 2 & & & & & 1 & 0 & 1 & & 1 & 0 \\
\hline 5 & 7 & 4 & & & & & 1 & 1 & 1 & 1 & 0 & 0 \\
\hline 6 & 11 & 2 & & & & 1 & 0 & 1 & 1 & & 1 & 0 \\
\hline 7 & 13 & 4 & & & & 1 & 1 & 0 & 1 & 1 & 0 & 0 \\
\hline 8 & 17 & 2 & & & 1 & 0 & 0 & 0 & 1 & & 1 & 0 \\
\hline 9 & 19 & 4 & & & 1 & 0 & 0 & 1 & 1 & 1 & 0 & 0 \\
\hline 10 & 23 & 6 & & & 1 & 0 & 1 & 1 & 1 & 1 & 1 & 0 \\
\hline 11 & 29 & 2 & & & 1 & 1 & 1 & 0 & 1 & & 1 & 0 \\
\hline 12 & 31 & 6 & & & 1 & 1 & 1 & 1 & 1 & 1 & 1 & 0 \\
\hline 13 & 37 & 4 & & 1 & 0 & 0 & 1 & 0 & 1 & 1 & 0 & 0 \\
\hline 14 & 41 & 2 & & 1 & 0 & 1 & 0 & 0 & 1 & & 1 & 0 \\
\hline 15 & 43 & 4 & & 1 & 0 & 1 & 0 & 1 & 1 & 1 & 0 & 0 \\
\hline 16 & 47 & 6 & & 1 & 0 & 1 & 1 & 1 & 1 & 1 & 1 & 0 \\
\hline 17 & 53 & 6 & & 1 & 1 & 0 & 1 & 0 & 1 & 1 & 1 & 0 \\
\hline 18 & 59 & 2 & & 1 & 1 & 1 & 0 & 1 & 1 & & 1 & 0 \\
\hline 19 & 61 & 6 & & 1 & 1 & 1 & 1 & 0 & 1 & 1 & 1 & 0 \\
\hline 20 & 67 & 4 & 1 & 0 & 0 & 0 & 0 & 1 & 1 & 1 & 0 & 0 \\
\hline
\end{tabular}


- для положительных простых чисел

$z_{2}=\frac{1}{2}-\frac{1}{2} \sin \left(\frac{\pi}{2} P_{Z=3, \ldots}\right) ;$

- для отрицательных простых чисел

$$
z_{2}=-\frac{1}{2}-\frac{1}{2} \sin \left(\frac{\pi}{2} P_{Z=-3, \ldots}\right) .
$$

Таким образом, гипотеза Римана о корне 1/2 доказывается и на ряде ЦПЧ.

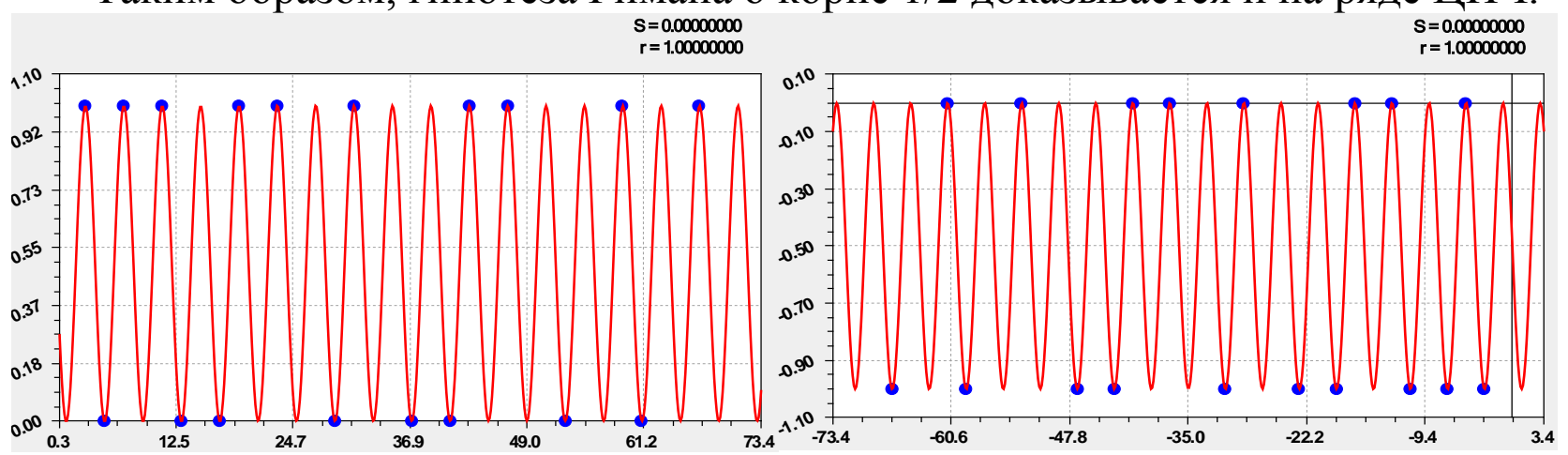

график по модели (19) положительных ПЧ график по модели (20) отрицательных ПЧ

Рисунок 9. Распределение двоичного числа по правой и левой некритическом ЦПЧ

С ростом разряда числа $\pi$ более 18 [9,10] остатки будут меньше 1е-12.

Влияние ЦПЧ в симметричном ряду $P_{Z 20}$ на двоичное число $z_{2}$ части прироста по вертикали $i_{Z}^{p}=2$ определяется эмпирической формулой

$$
z_{2}=0,5-0,5 \sin \left(\pi P_{Z 20} / 1,73942\right) .
$$

Затем пришлось исключить шесть значений ЦПЧ $(-3,-2,-1,0,1,2)$ из-за наличия тривиальных нулей. Положительный ряд ПЧ начинается с 3 . А отрицательный ряд ПЧ начинается с цифры -5 , поэтому даже в симметричном ряду ЦПЧ наблюдается асимметрия в двоичной квантификации прироста.

Влияние прироста ЦПЧ (ряд $p_{Z 20}$ ) на двоичное $z_{2}$ по вертикали $i_{Z}^{p}=2$ :

$$
z_{2}=0,5-0,5 \cos \left(\pi p_{Z 20} / 2,12144\right) \text {. }
$$

Из-за исключения шести точек влияние прироста на вторую вертикаль двоичного представления получается модель (рис. 10) вида

$$
z_{2}=0,5-0,5 \cos \left(\pi p_{z 20-6} / 2\right) .
$$

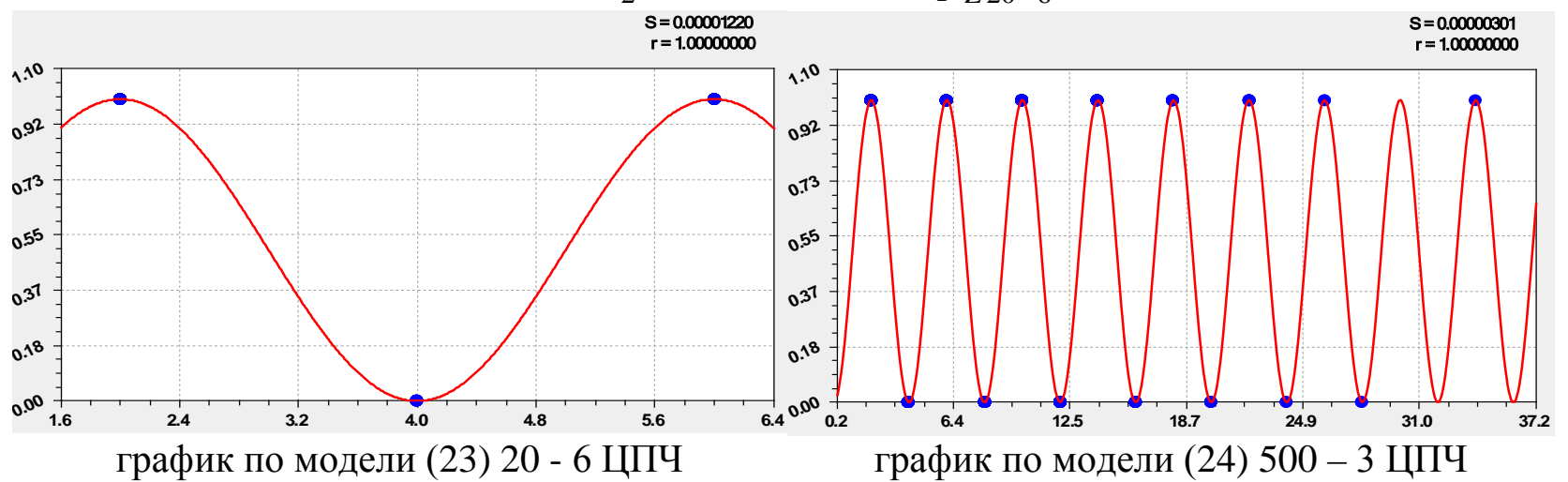

Рисунок 10. Распределение двоичного числа некритических ЦПЧ от прироста ЦПЧ

Для массива из $500-3=497$ некритических ПЧ (рис. 10) дана формула 


$$
z_{2}=0,5-0,5 \cos \left(\pi P_{Z \pm(500-3)} / 2\right) .
$$

Таким образом, гипотеза Римана доказана и на ряду целых простых чисел.

Блочная структура ряда ЦПЧ. В любом конечномерном ряду простых чисел, включая и целые простые числа, блоки выделяются через преобразование, то есть квантование, простых чисел из десятичной в двоичной системе счисления. Разделим ряд ЦПЧ по блокам (разрядам $i$ ) двоичного счисления (табл. 3) до мощности по возможностям программной среды CurveExpert-1.40.

Таблица 3

Длина, левая м правая граница блоков целых простых чисел

\begin{tabular}{|c|c|c|c|c|c|c|c|c|c|c|}
\hline \multirow[b]{2}{*}{$\begin{array}{l}\text { Разряд } \\
i\end{array}$} & \multirow[b]{2}{*}{ $\pm 2^{i-1}$} & \multicolumn{3}{|c|}{ Левая граница } & \multicolumn{3}{|c|}{ Правая граница } & \multicolumn{3}{|c|}{ Длина блока двоичных чисел } \\
\hline & & $\pm P_{N}^{\prime}$ & $n_{ \pm}^{\prime}$, шт. & $2 P_{N}^{\prime} /\left(n_{ \pm}^{\prime}-1\right)$ & $\pm P_{N}^{\prime \prime}$ & $n_{ \pm}^{\prime \prime}$, шт. & $2 P_{N}^{\prime \prime} /\left(n_{ \pm}^{\prime \prime}-1\right)$ & $\Delta P_{N}=P^{\prime \prime}-P$ & $\Delta n=n^{\prime \prime}-n^{\prime}$ & $2 \Delta P_{N} / \Delta n$ \\
\hline 0 & $1 / 2$ & $1 / 2$ & 1 & - & $1 / 2$ & 1 & - & 0 & 0 & - \\
\hline 1 & 1 & 1 & 3 & 1 & 1 & 3 & 1 & 0 & 0 & - \\
\hline 2 & 2 & 2 & 5 & 1 & 2 & 5 & 1 & 0 & 0 & - \\
\hline 3 & 4 & 3 & 7 & 1 & 3 & 7 & 1 & 0 & 0 & - \\
\hline 4 & 8 & 5 & 9 & 1,2500 & 7 & 11 & 1,4000 & 2 & 2 & 2 \\
\hline 5 & 16 & 11 & 13 & 1,8333 & 13 & 15 & 1,8571 & 2 & 2 & 2 \\
\hline 6 & 32 & 17 & 17 & 2,1250 & 31 & 25 & 2,5833 & 14 & 8 & 3,5000 \\
\hline 7 & 64 & 37 & 27 & 2,8462 & 61 & 39 & 3,2105 & 24 & 12 & 4,0000 \\
\hline 8 & 128 & 67 & 41 & 3,3500 & 127 & 65 & 3,9688 & 60 & 24 & 5,0000 \\
\hline 9 & 256 & 131 & 67 & 3,9697 & 251 & 111 & 4,5636 & 120 & 44 & 5,4545 \\
\hline 10 & 512 & 257 & 113 & 4,5893 & 509 & 197 & 5,1939 & 252 & 84 & 6,0000 \\
\hline 11 & 1024 & 521 & 199 & 5,2626 & 1021 & 347 & 5,9017 & 500 & 148 & 6,7568 \\
\hline 12 & 2048 & 1031 & 349 & 5,9253 & 2039 & 621 & 6,5774 & 1008 & 272 & 7,4118 \\
\hline 13 & 4096 & 2053 & 623 & 6,6013 & 4093 & 1131 & 7,2442 & 2040 & 508 & 8,0315 \\
\hline 14 & 8192 & 4099 & 1133 & 7,2420 & 8191 & 2059 & 7,9602 & 4092 & 926 & 8,8380 \\
\hline
\end{tabular}

Все параметры из таблицы 3 идентифицируются законом показательного роста. Корень $1 / 2$ автоматически получается при условии $i=0$. Граница между блоками простых чисел точно определяется соответствующей частью из десятичной системы счисления. Именно здесь происходят не понятные математикам скачки второго типа (первый тип скачка находится внутри блоков по так называемым первичным приростам) в ряду у смежных простых чисел.

Эту границу приближенно можно заменить (рис. 11) уравнением

$$
\pm 2^{i-1} \approx 1 / 2+5,04767 \cdot 10^{-7} i^{8,90632} \text {. }
$$

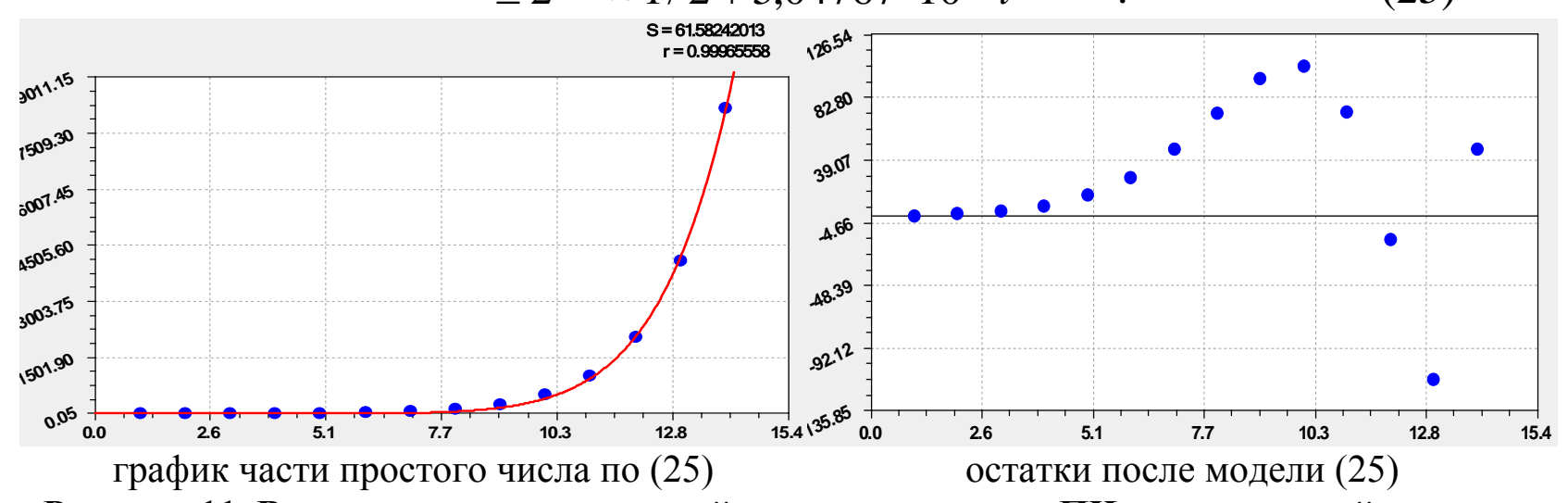

Рисунок 11. Влияние разряда двоичной системы на часть ПЧ из десятичной системы 
Каждый блок ПЧ $[2,4]$ определяется левой и правой границами, а также длиной блока вдоль ряда простых чисел. У всех формул остатки по виду схожи с точками на рисунке 11.

Для сравнения на рисунке 12 даны графики левой и правой границы.

Сложнее средние у ПЧ до левой и правой границы блоков (рис. 13).

Вначале по трендам от разряда двоичной системы получены формулы:

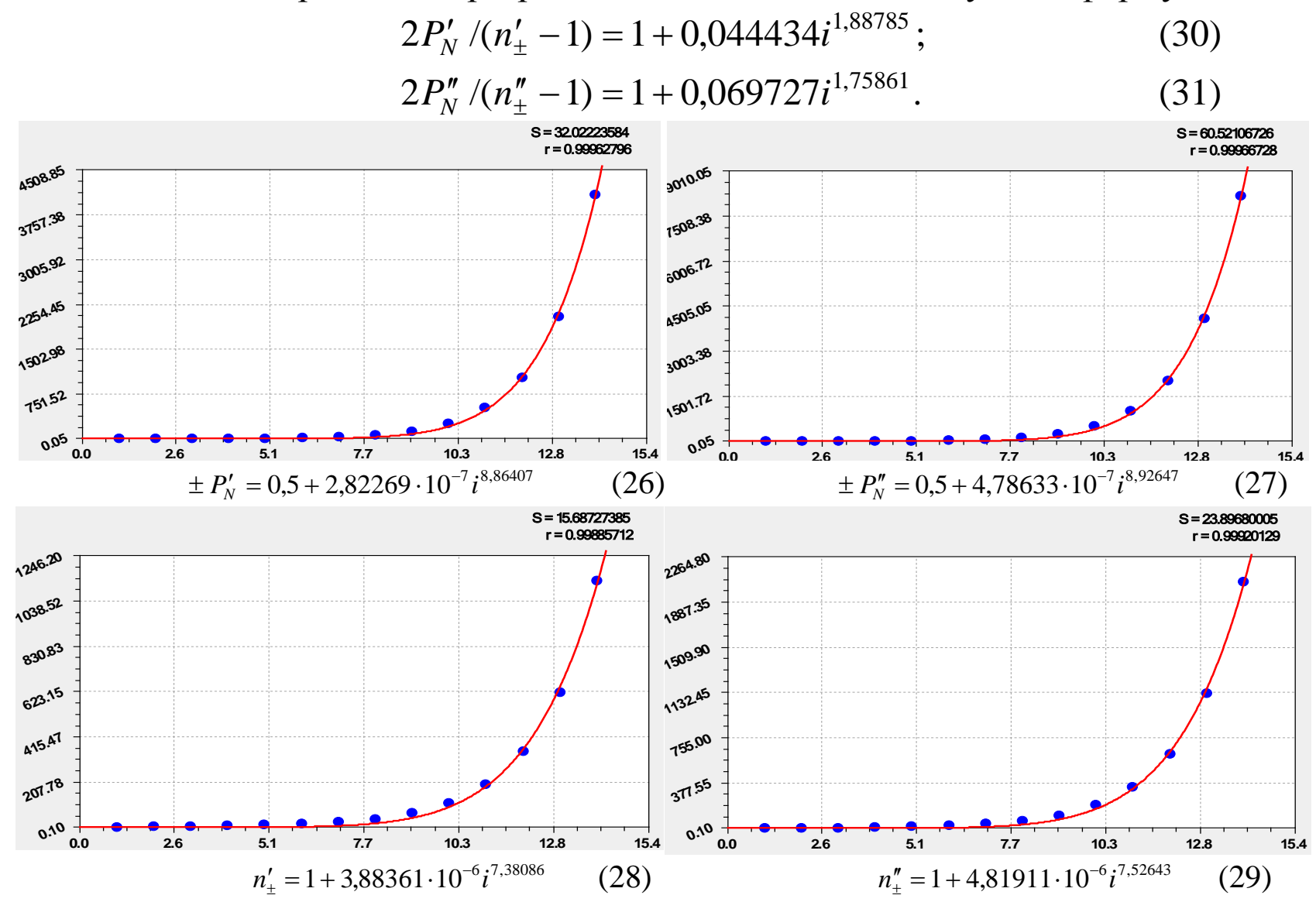

Рисунок 12. Параметры левой и правой границы блоков простых чисел

Затем произошло дополнение тренда простой синусоидой по выражениям:

$$
\begin{aligned}
& 2 P_{N}^{\prime} /\left(n_{ \pm}^{\prime}-1\right)=1+0,043455 i^{1,88851}-0,26761 \sin (\pi i / 6,68984) ; \\
& 2 P_{N}^{\prime \prime} /\left(n_{ \pm}^{\prime \prime}-1\right)=1+0,060123 i^{1,81554}-0,35172 \sin (\pi i / 6,18813) .
\end{aligned}
$$

Остатки после формул (32) и (33) показывают возможность дополнительной волновой функции со снижающейся амплитудой. Как видно из данных таблицы 3 , длина блока начинается только с $i=4$. Поэтому простые числа начинают упорядочиваться не с центра симметрии, а гораздо дальше от него.

Для трех параметров (без учета до $i=3$ ) получены (рис. 14) уравнения:

$$
\begin{gathered}
\Delta P_{N}=P^{\prime \prime}-P^{\prime}=2,02671 \cdot 10^{-7} i^{8,98930} \\
\Delta n=n^{\prime \prime}-n^{\prime}=1,35859 \cdot 10^{-6} i^{7,70459} \\
2 \Delta P_{N} / \Delta n=-0,90145+0,69605 i
\end{gathered}
$$

Наилучшим параметром для оценки ряда простых чисел становится средняя длина блока двоичного представления целых простых чисел. Причем достаточно разложить только правую положительную сторону этого ряда. Как 
видно из рисунка 14 , остатки после $i \geq 10$ постепенно приближаются к нулю, и длина каждого блока в дальнейшем возрастает пропорционально разряду двоичной системы счисления. Тогда получается, что мощность ряда ПЧ и/или ЦПЧ удобнее считать по блокам, чем по наличию простых чисел в разрядах десятичной системы счисления. Это - еще один закон распределения простых чисел.

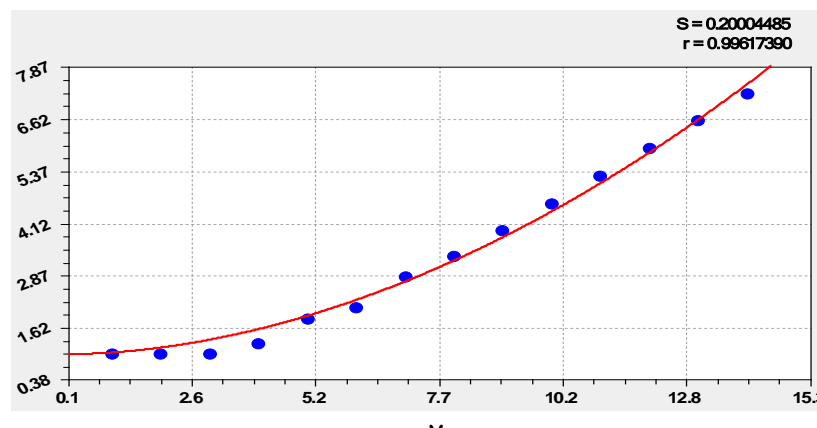

тренд левой границы
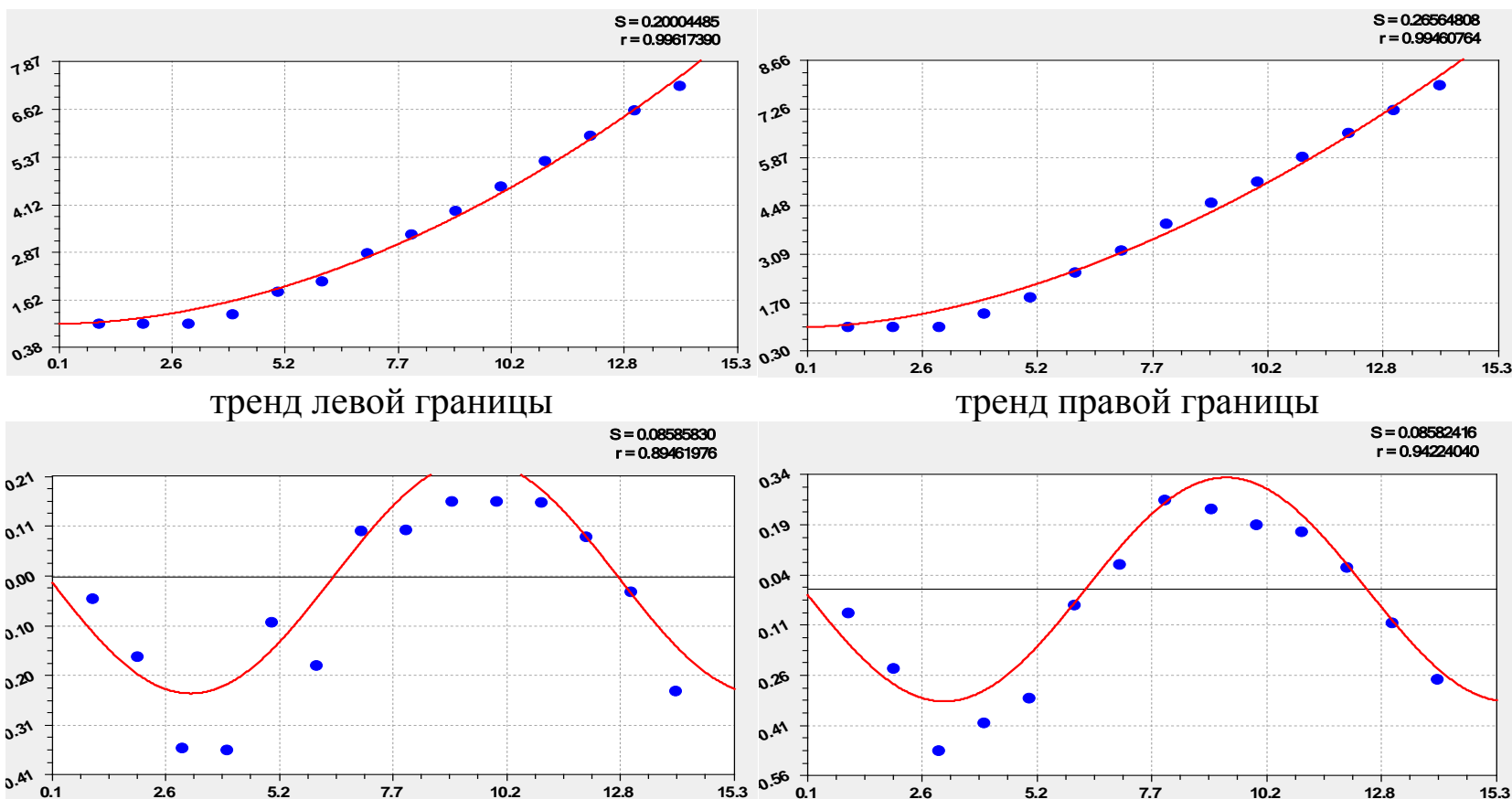

тренд правой границы

0.08585830

волновое возмущение границы блоков $S=0.07476915$ $\mathrm{r}=0.9995553$

волновое возмущение границы блоков
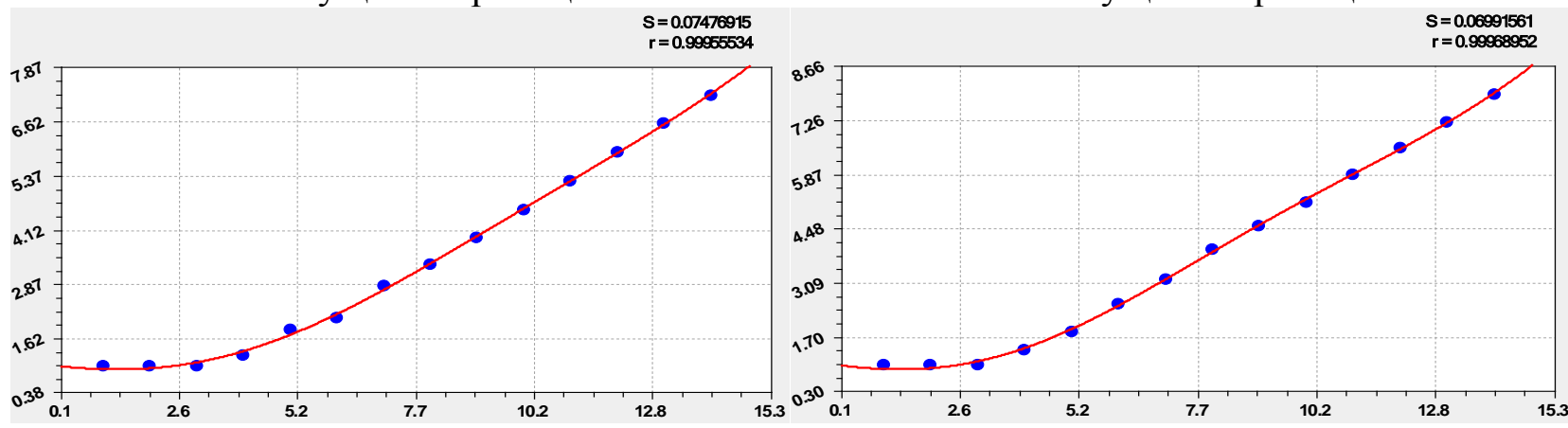

график модели (32)

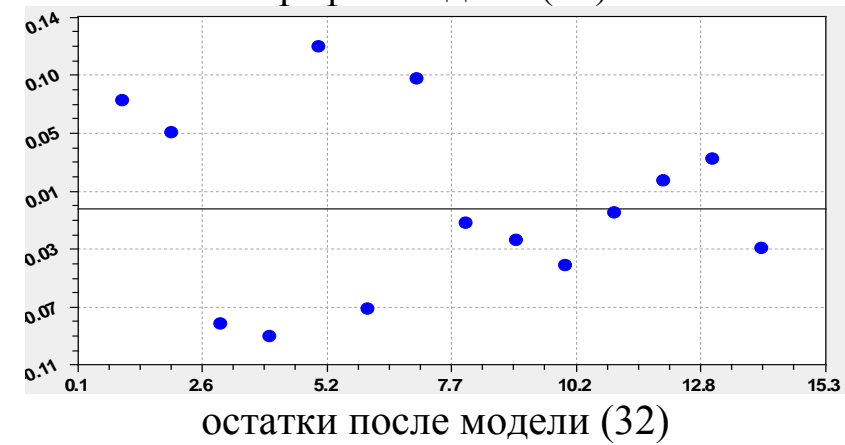

график модели (33)

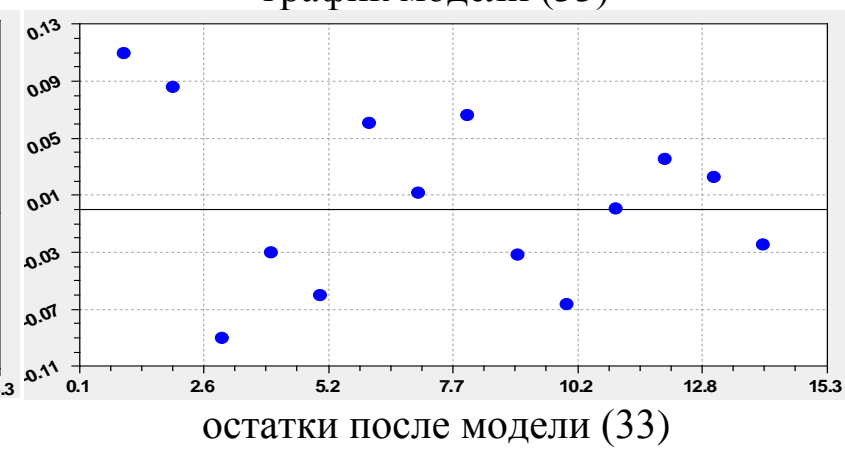

Рисунок 13. Средние интервалы ПЧ на границах блоков двоичного разложения ЦПЧ

Вещественная часть 1/2. Из Интернет известно: «А вот знаменитая гипотеза Римана, что вещественная часть корня всегда в точности равна $1 / 2$, ещё никем не доказана, хотя её доказательство имело бы для теории простых чисел в высшей степени важное значение». 
Уравнение (3) и его частные случаи доказывают, что не только вещественная часть корня равна $1 / 2$.

Есть и другие интересные математические результаты. Например, в формулах (5) и (7) выражение перед функцией косинуса также точно равно 1/2. Появление числа пространства $\pi$ превращает уравнение (5) в сигнал в виде симметричного вейвлета с постоянной амплитудой $\pm 1 / 2$.

Дзета-функции Римана имеет нули в отрицательных числах, кратных 2 . Но данные таблицы 1 показывают, что кратность равна $2^{i_{2}-1}$.

Тогда по Риману получается, что $2^{i_{2}-1}=2$ только при условии $i_{2}=2$.
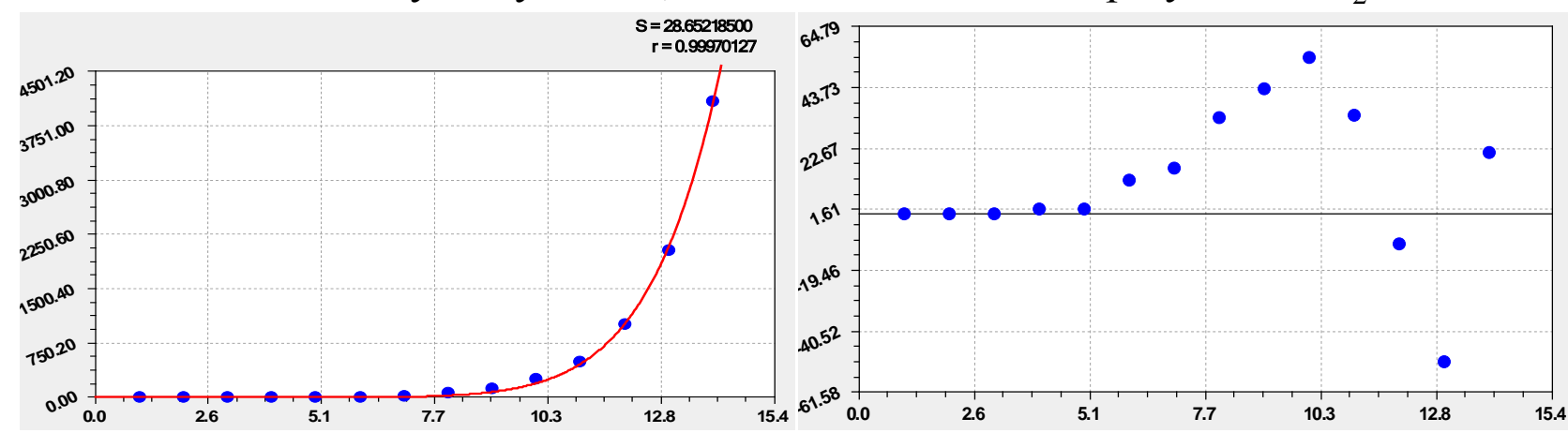

длина блока двоичных чисел

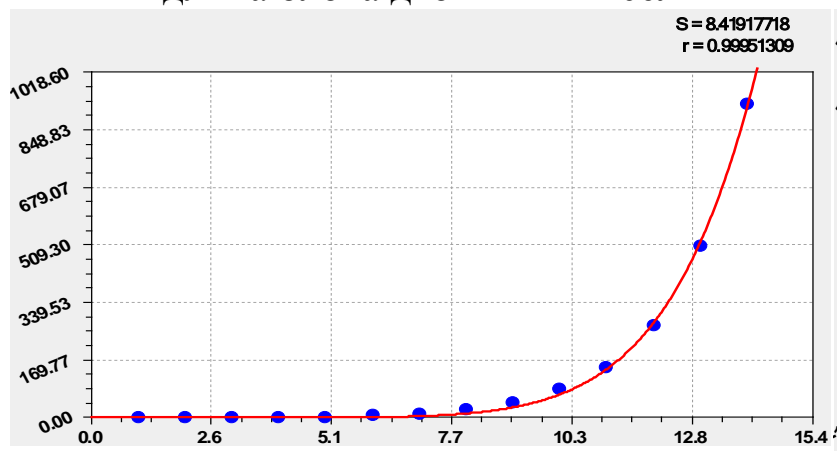

количество простых чисел в блоке

остатки после модели (34)
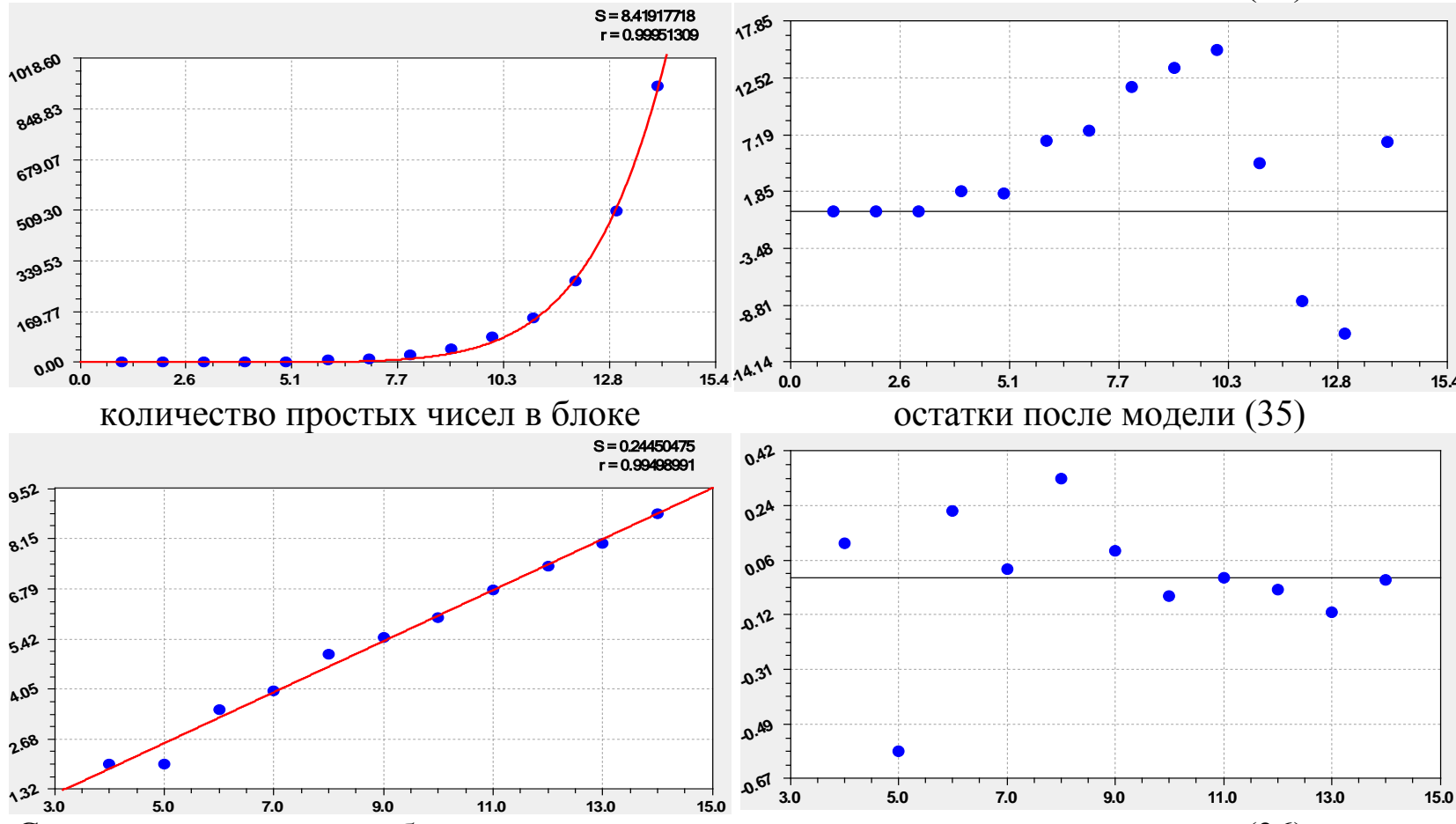

Средняя длина каждого блока простых чисел

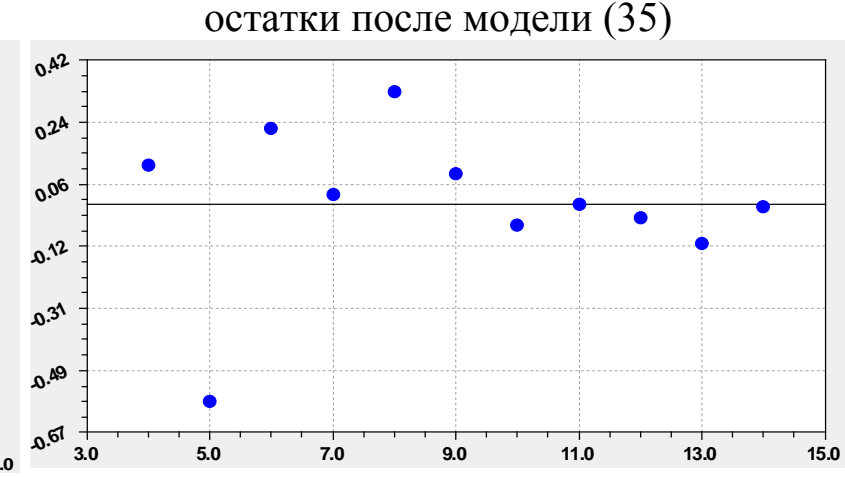

остатки после модели (36)

Рисунок 14. Длина блоков у двоичного представления ряда простых чисел

Заметим также, что в дзета-функции Римана в комплексных переменных принята функция синуса, но косинус лучше для действительных чисел, так как позволяет не обращать внимания на знаки в выражении под тригонометрической функцией. Косинус работает в обоих квадрантах на ряде натуральных чисел $(0,1,2, \ldots, \infty)$. Поэтому эта тригонометрическая функция будет удачной и дальше в изучении рядов простых чисел. 
Заключение. Увлечение факторизацией не позволило математикам применить двоичную систему для анализа не только множителей любого простого числа, но и квантованных в двоичной системе счисления слагаемых от любого натурального и простого числа. Разложение по слагаемым из двоичной системы счисления дает преимущества по наглядности.

Для бесконечномерного ряда простых чисел имеем уровни квантования или область разрядов двоичной системы. В итоге получаем два типа нулей тривиальные и нетривиальные. Первые расположены слева до вертикали в каждом блоке в виде единицы. Нетривиальные нули располагаются внутри двух столбцов с единицами, причем левый столбец 1 сдвигается по блокам справа налево по мере увеличения простого числа.

Разряд простого числа двоичной системы счисления играет решающую роль для разложения известного простого числа и прогнозирования очередного неизвестного простого числа.

Сравнение показало существенность в блоках только самой последовательности простых чисел, а порядковый номер имеет только вспомогательное значение. Полнота порядкового номера будет соблюдена только при принятии полного ряда $\mathrm{N}=\{0,1,2,3,4,5,6, \ldots\}$ натуральных чисел. Нуль нельзя игнорировать на оси абсцисс. Поэтому основным показателем становится среднестатистическое (только не среднеарифметическое) двоичное число, а переменной само простое число. Последовательно рассматривая все блоки ряда простых чисел доказано, что для первого столбца (правая граница двоичной записи простых чисел) условие $z_{2}=1$ будет соблюдаться на всей бесконечной полуоси простых чисел некритичного ряда. Левая граница будет смещаться скачкообразно от блока к блоку по мере роста значения простого числа.

По мере роста разряда двоичной системы счисления поперек ряда в блоке № 11 из традиционного ряда $a(n)=(1031,2039)$ простых чисел происходит снижение адекватности статистической модели.

\section{Список литературы:}

1. P.M. Mazurkin, "Wavelet Analysis of a Number of Prime Numbers." American Journal of Numerical Analysis, vol. 2, no. 2 (2014): 29-34. doi: 10.12691/ajna-2-2-1.

2. P.M. Mazurkin. Patterns of primes. Germany: Palmarium Academic Publishing, 2012. 280 p.

3. P.M. Mazurkin, "Stable Laws and the Number of Ordinary." Applied Mathematics and Physics, vol. 2, no. 2 (2014): 27-32. doi: 10.12691/amp-2-2-1.

4. P.M. Mazurkin, "Series Primes in Binary." American Journal of Applied Mathematics and Statistics, vol. 2, no. 2 (2014): 60-65. doi: 10.12691/ajams-2-2-2.

5. P.M. Mazurkin, "Proof the Riemann Hypothesis." American Journal of Applied Mathematics and Statistics, vol. 2, no. 1 (2014): 53-59. doi: 10.12691/ajams-2-2-1.

6. P.M. Mazurkin, "Increment Primes." American Journal of Applied Mathematics and Statistics, vol. 2, no. 2 (2014): 66-72. doi: 10.12691/ajams-2-2-3.

7. P.M. Mazurkin, "Block Structure of a Number of the Integers Prime." Applied Mathematics and Physics, vol. 2, no. 4 (2014): 135-145. doi: 10.12691/amp-2-4-3.

8. P.M. Mazurkin, "Chaos and Order in the Integers Primes." Applied Mathematics and Physics, vol. 2, no. 4 (2014): 146-156. doi: 10.12691/amp-2-4-4. 
9. P.M. Mazurkin. Statistical modeling of entire prime numbers / International Journal of Engineering and Technical Research (IJETR) ISSN: 2321-0869, Volume-2, Issue-8, August 2014. P.148158.

10. P.M. Mazurkin. Method of identification $/ / 14^{\text {th }}$ International multidisciplinary scientific geoconferenct \& SGEM2014. GeoConference jn NANO, BIO AND GREEN - TECHNOLOGIES FOR A SUSTAINABLE FUTURE. Conference proceedincs. Volume 1. Section Advances in Biotechnology. 17-26 June 2014. Albena, Bulgaria. P. 427-434. 